\title{
Psiquiatria no século XXI: transformações a partir da integração com a Atenção Primária pelo matriciamento
}

I ${ }^{1}$ Sandra Fortes, ${ }^{2}$ Alice Menezes, ${ }^{3}$ Karen Athié, ${ }^{4}$ Luiz Fernando Chazan, ${ }^{5}$ Helio Rocha, ${ }^{6}$ Joana Thiesen, ${ }^{7}$ Celina Ragoni, ${ }^{8}$ Thiago Pithon, ${ }^{9}$ Angela Machado I

Resumo: A lacuna de cuidados em Saúde Mental (SM) é um problema mundial. A Atenção Primária à Saúde (APS) é a base para estruturação da rede assistencial integral que o solucione. Sua integração com a Psiquiatria é parte deste desafio. Partindo da pergunta norteadora "Como vem sendo desenvolvida a inserção da APS no cuidado em Saúde Mental através da integração com a Psiquiatria pelo Matriciamento?”, este artigo analisa a participação da Psiquiatria na organização da assistência em saúde mental centrada na APS, dentro de um modelo integral de cuidado em saúde. Foi utilizada metodologia qualitativa baseada na problematização da integração do Psiquiatra com as equipes da Estratégia de Saúde da Família no processo de matriciamento, sistematizam-se as experiências e conhecimento desenvolvidos pelos autores nos projetos do Laboratório Interdisciplinar de Pesquisa em APS da Universidade do Estado do Rio de Janeiro. A partir dos referenciais conceituais dos Cuidados Colaborativos, Apoio Matricial, Matriciamento e Cuidado Integral, e da caracterização das demandas clínicas de SM mais frequentes na APS, discutem-se as repercussões destes na estruturação do cuidado em SM, na formação médica e na transformação da Psiquiatria. Conclui-se que a APS é um novo campo de atuação da Psiquiatria trazendo desafios importantes para a formação e a assistência em SM.

> Palavras-chave: Saúde Mental; Psiquiatria; Atenção Primária; matriciamento; integralidade.

\author{
1 Psiquiatra, professora adjunta, \\ Faculdade de Ciências Médicas (FCM- \\ UERJ); coordenadora do Laboratório \\ Interdisciplinar de Atenção Primária \\ à Saúde (LIPAPS), Universidade do \\ Estado do Rio de Janeiro (UERJ). \\ Rio de Janeiro-RJ, Brasil. Endereço \\ eletrônico: sandrafortes@gmail.com \\ 2 Psicóloga, doutoranda em Saúde \\ Coletiva no IMS-UERJ, com bolsa \\ CNPQ, e LIPAPS-UERJ. Rio de \\ Janeiro-RJ, Brasil. Endereço eletrônico: \\ menezes_alice@yahoo.com.br \\ ${ }^{3}$ Psicóloga, doutoranda em Ciências \\ Médicas no Programa de Pós- \\ graduação em Ciências Médicas, \\ com bolsa CAPES, e LIPAPS-UERJ. \\ Rio de Janeiro-RJ, Brasil. Endereço \\ eletrônico: karensathie@gmail.com \\ ${ }^{4}$ Psiquiatra, professor assistente e \\ doutorando em Ciências Médicas, \\ Programa de Pós-graduação em \\ Ciências Médicas, Faculdade de \\ Ciências Médicas (FCM-UERJ) e LIPAPS- \\ UERJ. Rio de Janeiro-RJ, Brasil. Endereço \\ eletrônico: Ifchazan@gmail.com \\ ${ }^{5}$ Psiquiatra, LIPAPS-UERJ. Rio \\ de Janeiro-RJ, Brasil. Endereço \\ eletrônico: hearoch@gmail.com \\ ${ }^{6}$ Psiquiatra, LIPAPS-UERJ. Rio \\ de Janeiro-RJ, Brasil. Endereço \\ eletrônico: jothiesen@yahoo.com.br \\ ${ }^{7}$ Psiquiatra, LIPAPS-UERJ. Rio de \\ Janeiro-RJ, Brasil. Endereço eletrônico: \\ celinaragoni@yahoo.com.br \\ ${ }^{8}$ Psicólogo, consultor e apoiador \\ institucional da Organização Pan- \\ Americana de Saúde/Ministério da \\ Saúde, Brasília-DF; e LIPAPS-UERJ. \\ Rio de Janeiro-RJ, Brasil. Endereço \\ eletrônico: tmpithon@yahoo.com.br \\ ${ }^{9}$ Psicóloga, pós-doutoranda em \\ Saúde Coletiva no IMS-UERJ com \\ bolsa CNPQ, e LIPAPS-UERJ. Rio de \\ Janeiro-RJ, Brasil. Endereço eletrônico: \\ angelamachado@bighost.com.br
}


Em 1978, a Declaração Alma-Ata ${ }^{1}$ alertou para a necessidade de rever a organização dos sistemas de saúde no mundo, redirecionando-os para a Atenção Primária (APS), valorizando a assistência no território, com incentivo à promoção da saúde e prevenção de doenças. No Brasil, a reestruturação da APS ocorreu com a implantação da Estratégia de Saúde da Família (ESF), iniciada nos anos 90 e atualmente base do Sistema Único de Saúde (SUS).

Consoante com o que Starfield (2002) elenca como atributos inerentes à APS, a ESF é hoje importante porta de entrada no sistema de saúde, resolvendo $80 \%$ dos agravos, provendo atenção longitudinal, organizando o cuidado e ampliando a oferta de acesso à saúde (LAVRAS, 2011). Cada equipe da ESF responde por 3.500 pessoas adscritas e acompanhadas por médicos, enfermeiros e agentes comunitários (ACS), que têm a responsabilidade sanitária do cuidado no território. A ESF cuida desde pré-natal e puericultura até cuidados paliativos, previne e trata doenças crônicas como diabetes, hipertensão, Aids e transtornos mentais, e é procurada em situações de crise pessoais, relacionais e familiares. Esse cuidado abrange o acompanhamento dos casos mais graves em conjunto com os serviços especializados onde os profissionais da APS atuam na posição de profissionais generalistas. Essa nova organização transforma o funcionamento do sistema de saúde e atinge também a função e o processo de trabalho dos especialistas, exigindo sua integração com outros profissionais e níveis de assistência, não podendo se restringir a atuar isoladamente no seu núcleo específico de saber (CAMPOS, 1999).

Nesse processo também se incluem cuidados em saúde mental (SM), hoje uma prioridade na saúde pública mundial. $\mathrm{Na}$ ultima década, organismos como a Organização Mundial da Saúde - OMS (WHO, 2008) e grupos de profissionais e cientistas reunidos no Movement of Global Mental Health, ${ }^{2}$ com apoio do Lancet, têm ressaltado a importância da SM na saúde pública e a enorme lacuna de cuidado em SM existente no mundo de hoje, destacando a necessidade de acesso e transformação do modelo de atendimento em um modelo integral, biopsicossocial.

O mote lançado pela OMS, "No health without mental health" ou "Não há saúde sem saúde mental" (PRINCE et al., 2007), clama pela gravidade das 
necessidades de cuidado em SM e do "vazio assistencial". Aponta as altas taxas de falta de tratamento que variam entre $32,2 \%$ para a esquizofrenia e $78,1 \%$ para abuso e dependência de álcool, incluindo pelo menos 50\% daqueles com transtornos depressivo-ansiosos de vários subtipos. Essas taxas, mesmo variando por região, indicam um problema de saúde pública, colocando na pauta da OMS a necessidade de estudos e investimento para ampliar a cobertura e o acesso a intervenções em SM realizadas na APS por médicos generalistas no nível comunitário (WHO, 2005; 2011).

Qual o impacto para a Psiquiatria de sua integração com a APS através do cuidado colaborativo (matriciamento) com a ESF? Essa pergunta norteia este artigo cujo objetivo é analisar o papel da Psiquiatria nos sistemas assistenciais na APS baseados num modelo de cuidado integral à saúde. Inicialmente, serão apresentados os referenciais conceituais da integração entre SM e APS, passando pela definição de Cuidados Colaborativos, Apoio Matricial e Matriciamento. Em seguida, serão caracterizadas as demandas de SM mais frequentes na APS: pessoas com transtornos mentais comuns, transtornos mentais graves e persistentes, uso nocivo de álcool e a família como objetos do cuidado compartilhado entre a ESF e o psiquiatra-matriciador. Por fim, serão discutidos repercussões e limites do novo campo de atuação da Psiquiatria.

Utilizou-se metodologia qualitativa baseada na problematização. Neste sentido, buscou-se sistematizar o conhecimento empírico desenvolvido no processo de construção de soluções para os problemas concretos encontrados na realidade social, ou seja, as necessidades e demandas de cuidado de SM na APS. Assim, recorreu-se à revisão da literatura para fundamentar as observações registradas durante participação dos autores em ações de matriciamento realizadas ao longo dos projetos do Laboratório Interdisciplinar de Pesquisa em Atenção Primária à Saúde (LIPAPS/UERJ).

Fundado em 2010, o LIPAPS/UERJ surgiu a partir de várias atividades, projetos e publicações nas áreas de extensão, ensino e pesquisa desenvolvidas, desde 2002, pelo Grupo de Pesquisa CNPq "Saúde Mental na Atenção Primária", da Faculdade de Ciências Médicas da Universidade do Estado do Rio de Janeiro (FCM/UERJ), e desde então - tem o compromisso de ajudar a construir o cuidado de SM na APS. 


\section{A integração entre a Saúde Mental e a Atenção Primária: cuidados colaborativos, apoio matricial e matriciamento}

A integração dos psiquiatras com a APS envolve modificar a relação entre estes profissionais e a organização do fluxo de usuários entre o nível assistencial primário e o especializado. Tradicionalmente, esse fluxo ocorre através das referências/encaminhamentos com a transferência da responsabilidade do cuidado para o médico especialista sem manutenção de uma comunicação entre os profissionais. Com a atuação da ESF na resolução, prevenção e reabilitação em SM, faz-se necessário uma nova forma de articulação entre os dois níveis. Este é um desafio para o mundo inteiro que tem construído diferentes propostas de colaboração e compartilhamento do cuidado em coparticipação de generalistas e especialistas. No Reino Unido, a literatura refere experiências da inserção de um profissional, que pode ter diversas formações de base, denominado case manager, como apoio ao paciente, favorecendo acesso, garantindo a longitudinalidade do cuidado e a integração entre profissionais e serviços (BOWER et al., 2006; CHEW-GRAHAM et al., 2014; GASK et al., 2012; KNOWLES et al., 2013).

No Brasil, o modelo de cuidados colaborativos é o do Apoio Matricial desenvolvido por Gastão Wagner Campos e colaboradores em Campinas no final do século XX (CAMPOS, 1999), com diversas experiências no país ocorrendo geralmente, mas não exclusivamente, a partir dos Centros de Atenção Psicossocial - CAPS (LANCETTI, 2000). Ele se propõe a ser uma nova forma de relação entre a atenção primária e a atenção especializada, através de uma matriz de interação de diferentes saberes. Em 2008, a publicação da Portaria $n^{\circ}$ 154, que regula a criação dos Núcleos de Apoio a Saúde da Família (NASF) com a recomendação de haver pelo menos um profissional de SM, normatizou a prática de matriciamento, definindo-a como modelo de cuidados colaborativos no SUS brasileiro.

Diferentes modalidades de matriciamento vêm sendo desenvolvidas (ATHIE et al., 2013; MACHADO; CAMATA, 2013; BOMFIM, 2013), mas uma discussão mais profunda foge aos objetivos deste artigo. Aqui, o termo "matriciamento" será definido como: "atividade de atendimento colaborativo interdisciplinar onde um profissional especializado realiza um atendimento conjunto ou discussão de caso com a equipe de referência para traçar um plano terapêutico" (FORTES; BALLESTER, 2012, p. 266). 
Assim sendo, a proposta do NASF é trabalhar interdisciplinarmente em

conjunto com a APS, em ações de interconsulta, discussão de casos, consultas conjuntas e visitas domiciliares para caracterização dos problemas específicos de sua demanda, desenvolvimento de novos tipos de intervenção terapêutica, estruturação da rede de cuidado em saúde e de parcerias intersetoriais com os recursos comunitários. $\mathrm{O}$ matriciamento tem caráter clínico, no que se refere ao cuidado com o usuário; de estruturação da rede de cuidados a partir da análise do contexto territorial, incluindo os recursos comunitários e da rede de cuidados, e de educação permanente, no que se refere às trocas de conhecimento realizadas entre o especialista e o generalista sobre suas diversas ações. Há uma ampliação da atenção em SM que modifica tanto a prática dos médicos de família quanto dos psiquiatras. O campo de trabalho do matriciamento é o campo da comunidade, da integralidade, da promoção de saúde e da prevenção dos transtornos, do trabalho com grupos, das ações interdisciplinares e intersetoriais. Este novo campo de ação da psiquiatria não só inclui a neurociência (base da formação biomédica dos psiquiatras) como, por se estruturar dentro da abordagem biopsicossocial, também atua sobre os determinantes sociais da saúde, estimula a promoção da saúde, amplia o acesso, reduz as barreiras ao tratamento, combate o estigma e fortalece os direitos humanos. $\mathrm{O}$ compartilhamento de saberes amplia a visão e a compreensão de ambos os profissionais, aumentando sua autonomia e empoderamento para atuar dentro de uma proposta de abordagem integral dos problemas.

Assim sendo, é possível dizer que o NASF, o apoio matricial e o matriciamento são os novos dispositivos, campo e processo de trabalho para a Psiquiatria no século XXI, abrindo perspectivas, transformações e desafios na sua prática, bem como na sua formação.

\section{A Psiquiatria e a abordagem integral}

A formação médica tradicional baseia-se no modelo biomédico e na abordagem centrada na doença, e o desenvolvimento de uma compreensão integral, biopsicossocial, como preconizada pelas novas diretrizes curriculares (BRASIL, 2014) é um grande desafio. A formação dos médicos de família e comunidade (MFC) tem a integralidade como pressuposto básico e a abordagem centrada na pessoa como proposta de trabalho, construindo correlações entre o sofrimento 
psíquico e físico. Assim, o entendimento do paciente sobre seu adoecimento é tão importante quanto o entendimento do processo patológico, como bem definido por Kleinman (1988) nos conceitos de illness (sofrimento - que guarda relação com a experiência de mal-estar do paciente) e disease (doença - que se refere às anormalidades em órgãos e sistemas avaliadas pelos médicos). Rompendo essas diferentes compreensões, médico e pessoa precisam construir uma comunicação que integre seus diferentes pontos de vista e estabeleça um plano terapêutico conjunto, com a participação e autonomia do usuário, construa o cuidado integral, como definido por Pinheiro (2014, s/p):

[...] a noção de "cuidado" integral permite inserir, no âmbito da saúde, as preocupações pelo bem estar dos indivíduos - opondo-se a uma visão meramente economicista - e devolver a esses indivíduos o poder de julgar quais são suas necessidades de saúde, situando-os assim como outros sujeitos e não como outros-objetos.

Nessa perspectiva, o apoio do psiquiatra matriciador às equipes representa também um espaço para reflexão sobre os aspectos psicossociais dos casos compartilhados, com troca de conhecimentos e construção de projetos terapêuticos singulares - PTS (OLIVEIRA, 2011). E pode envolver até questôes da relação médico-paciente por ser um espaço seguro para a elaboração das angústias da equipe sobre seu trabalho, incluindo os desconhecimentos, dificuldades, fantasias e preconceitos em relação ao sofrimento emocional, aos transtornos mentais e ao cuidado dos pacientes. Produz suporte social, que foi definido por Campos (2005), como fruto de relaçôes interpessoais grupais ou comunitárias que ofertam um ambiente vivenciado como apoiador e protetor, propiciando bem-estar psicológico e diminuição do estresse, contribuindo de forma extremamente efetiva para o suporte aos profissionais e a construção da Integralidade na tarefa assistencial.

\section{Reflexões sobre as demandas de Saúde Mental na Atenção Primária}

Nesta seção, são discutidas as demandas mais frequentemente para dirigidas ao psiquiatra durante as atividades de cuidado colaborativo com a APS, que são: a abordagem à família, os transtornos mentais comuns, os transtornos mentais graves e persistentes e o uso nocivo de álcool. 


\section{O matriciamento como espaço de cuidado à família}

Adotando o modelo integral e ampliando a atenção à saúde para uma ótica coletiva, a ESF considera a família seu núcleo básico de cuidado. Seus profissionais são referência para toda a família, pois cuidam de seus membros desde o pré-natal até o fim de suas vidas. Reconhecer o lugar da família como matriz da identidade (MINUCHIN, 1982) e como unidade primordial da rede social contribui para o desenvolvimento de estratégias que a insiram nos projetos terapêuticos e preventivos.

Esta abordagem é geralmente positiva, sendo prioritária nos casos de famílias de pacientes psicóticos; dependentes químicos; portadores de doenças crônicas com mau resultado no acompanhamento rotineiro; famílias com história de violência; aquelas com crianças ou adolescentes sem frequentar adequadamente a escola e as que vivenciam graves problemas sociais como miséria ou desemprego crônico. Dessa maneira se amplia a efetividade das ações de prevenção e tratamento na ESF (BIGRAS; MACHADO, 2014). Além desses, frequentemente, a ESF é solicitada a cuidar de famílias em situações de crise aguda, mesmo que não sejam famílias com graves problemas de relacionamento, pois esse trabalho inclui o fortalecimento da resiliência familiar. Dentre as estratégias para alcançar esse objetivo, podem ser utilizadas: saber identificar e confiar nas potencialidades e nos recursos familiares e envolver toda a família no processo de resolução de problemas individuais de seus membros (WALSH, 2005).

Apoiar essa abordagem e manejo é parte da atuação do psiquiatramatriciador na APS. Novos conhecimentos e práticas são necessários para estas intervenções, tais como entender a organização sistêmica da família, o ciclo de vida familiar, a dimensão triangular dos relacionamentos humanos, além da utilização de instrumentos como o Genograma e a Escala de Coelho (CHIAVERINI et al., 2011).

$\mathrm{Na}$ prática cotidiana do matriciamento, o comparecimento da família acompanhando o paciente é a oportunidade para que os objetivos mencionados sejam trabalhados. No matriciamento desenvolvido no Programa de Ensino e Trabalho do Laboratório de Habilidades Interpessoais e Sociais - PET LAHIS (BRASIL, 2010) na ESF, por alguns dos autores, observou-se que intervenções com dois ou três atendimentos compartilhados têm se mostrado suficientes para 
construir novas estratégias de enfrentamento das situações de crise. A colaboração do matriciador torna a equipe mais segura e ajuda no desenvolvimento de habilidades apontadas como importantes pela terapia familiar sistêmica (McDANIEL et al., 1994), tais como: saber engajar os membros da família, incluindo aqueles relutantes, numa reunião familiar conduzir essa reunião para que todos tenham oportunidade de expressão; ajudar os membros da família a verem suas dificuldades e como desenvolver novas formas de esforço cooperativo. Também é fundamental que o profissional seja capaz de identificar a disfunção familiar grave que exija encaminhamento para atendimento especializado.

Entretanto, é pouco comum encontrar profissionais da ESF e psiquiatras conhecedores dos processos sistêmicos que organizam e mantêm as famílias como um sistema saudável e que saibam utilizar esse conhecimento no espaço clínico da APS. Embora haja recomendação de treinamento de abordagem familiar nas residências em Psiquiatria (COELHO et al., 2005), isso ainda não ocorre, o que confirma a importância de repensar a formação do psiquiatra para a prática do matriciamento.

\section{O matriciamento como espaço de cuidado ao sofrimento emocional e aos transtornos mentais mais prevalentes na atenção primária}

$\mathrm{O}$ conceito de transtornos mentais comuns (TMC) refere-se a um modelo dimensional do sofrimento mental na comunidade ou na APS que engloba os transtornos depressivo-ansiosos e o sofrimento emocional difuso, que não preenchem critérios diagnósticos psiquiátricos e comprometem a qualidade de vida, demandando cuidado (GOLDBERG, 1992). Associam-se a determinantes sociais de gênero e étnico-raciais, iniquidades econômicas, e à presença de violência (ARAÚJO et al., 2005; FORTES et al., 2008, 2011; GONÇALVES; FORTES; TOFOLI, 2011). São observados coletivamente nas abordagens comunitárias, mas também individualmente no discurso de pacientes relatando problemas materiais, familiares e laborais. Esses fatores se potencializam, conforme verificado no aumento de três vezes do risco de TMC em mulheres que vivem em extrema pobreza (FORTES et al., 2011).

É importante reconhecer suas diferentes formas de manifestação e sua história singular. Na prática da APS, os TMC apresentam-se através de sintomas depressivos e ansiosos associados a queixas físicas inespecíficas, 
incluindo sintomas muito frequentes como irritabilidade, insônia, nervosismo, fadiga, esquecimento e falta de concentração. Representam a demanda de SM característica da APS e um desafio em especial no matriciamento. Longe de caracterizar uma psiquiatrização do sofrimento, cuidar deste sofrimento é reconhecer que a tristeza, a dor da perda, o medo, a irritação e certo nível de ansiedade são parte da vida de qualquer pessoa - logo, o adoecimento é indissociável da história singular, do contexto social e das condiçôes sóciodemográficas em que se vive (FONSECA; GUIMARÃES et al., 2008).

Os portadores desses transtornos têm na ESF sua principal porta de entrada e referência para o cuidado em saúde. Sua alta prevalência é alta, atingindo $25 \%$ da população comunitária no Brasil (MARAGNO et al., 2006) e mais de 50\% dos pacientes atendidos em unidades de APS (FORTES et al., 2008). Parcela significativa são transtornos depressivo-ansiosos, como demonstrado em estudo multicêntrico transversal que apontou frequência de depressão em torno de $25 \%$ e de ansiedade em torno de $37 \%$ entre usuários de quatro cidades brasileiras (GONÇALVES; BOWER, 2014).

Esse quadro clínico, geralmente, não faz as pessoas procurarem a assistência necessária e, quando o fazem, são subdiagnosticados e não recebem tratamento adequado. Isso é ainda mais relevante porque podem ocorrer comorbidades (exemplo: doenças como hipertensão e diabetes) que agravam o quadro clínico e levam à aderência inadequada aos tratamentos. Quando apresentam comprometimento funcional e da qualidade de vida, é importante tratar até casos leves e moderados, por possuírem alto risco de progressão para quadros mais severos (PORTUGAL et al., 2014; DEMYTTENAERE et al., 2004), observada especialmente nas populaçōes em regiōes de alta vulnerabilidade psicossocial com dificuldade histórica de acesso à assistência. Porém, estudos já demonstraram que as redes de suporte social construídas ao frequentar regularmente a igreja, participar de atividades esportivas e artísticas ou ter amigos ou parentes em quem se pode confiar, estão inversamente associadas à presença de TMC, por acionarem fatores de proteção (FORTES et al., 2011).

Avaliando-se os tratamentos recebidos, verificam-se diversos desafios para o psiquiatra matriciador. No estudo de Correia (2014), a proporção de mulheres com TMC de maior gravidade em tratamento farmacológico variava de apenas 12,4\% no Rio de Janeiro a 17,3\% em Porto Alegre, associado ao uso indiscriminado 
de benzodiazepínicos em detrimento do uso de antidepressivos, refletindo a baixa proporção e a fragilidade do tratamento destes casos (menos de $1 \mathrm{em}$ cada 5 casos). Sabe-se, porém, que esses pacientes apresentam melhoras quando apoiados pelas equipes da APS, individualmente ou em grupos (CHIAVERINI, 2011), o que justifica a possibilidade de desenvolvimento de outras intervenções terapêuticas além da medicação.

Além de diagnóstico e tratamento farmacológico adequado, é necessário abordar terapeuticamente os fatores psicossociais, oferecendo suporte para superação dos problemas individuais, familiares ou grupais, por meio do desenvolvimento de atividades coletivas e de integração aos espaços comunitários, buscando encontrar novas formas de enfrentamento e soluções para as dificuldades. É necessária a organização de cuidados escalonados de suporte onde a intensidade da intervenção é realizada de acordo com a severidade dos sintomas de SM, de modo que as abordagens farmacológicas e psicossociais são combinadas e ministradas em etapas, o que potencializa a resposta clínica das opções terapêuticas disponíveis e otimiza o uso de recursos escassos (COULL; MORRIS, 2011).

É imprescindível a inclusão de intervenções terapêuticas de suporte na carteira de alternativas de cuidado em SM a serem implementadas na ESF com o apoio do psiquiatra e outros profissionais de SM do NASF. O NASF, em conjunto com a ESF, pode organizar um sistema colaborativo de intervenções grupais que tornará possível produzir cuidados psicossociais de forma escalonada e progressiva que também incluam recursos de suporte comunitário. O diagnóstico de território é uma ferramenta estruturante para identificar os recursos a serem incorporados, através do trabalho intersetorial, no planejamento das ações de prevenção, promoção, tratamento e reabilitação em SM. Entretanto, essas abordagens são pouco citadas na literatura, sendo ainda desconhecidas pela maioria dos psiquiatras, que ao longo de sua formação e prática em geral dialogam apenas com modelos psicoterápicos como a psicanálise e a psicoterapia cognitivocomportamental. Embora estas sejam base teórica das intervenções desenvolvidas, assumem características técnicas e forma de implementação distintas na APS, sendo mais breves e estruturadas, incluindo planos de ação definidos e manuais de aplicação (MENEZES, 2012).

Analisando as intervençōes psicossociais para TMC na APS no mundo todo, verificou-se que existem desde intervenções com enquadre tradicional, com a 
presença de um profissional, até outras com arranjos diferenciados que envolvem a delegação de tarefas e o uso de mão de obra leiga em SM (task shifting), o suporte ao autocuidado, e aquelas mediadas por plataformas informatizadas (MENEZES, 2012). Presente em todo país, a Terapia Comunitária (TC) seria um modelo brasileiro deste tipo de intervenção, oferecendo espaço para as pessoas dividirem suas dores e sofrimentos. Pode ser conduzida por qualquer membro da equipe que possua formação adequada, sendo uma intervenção de promoção de SM e prevenção de agravamento dos quadros de sofrimento emocional a partir do enfrentamento de problemas em grupo (BARRETO, 2008). Ferreira Filho et al. (2009) apontaram alívio do sofrimento, valorização pessoal e fortalecimento dos vínculos como efeitos da TC, enquanto participantes de uma pesquisa sobre a TC no tratamento de depressão na ESF em Petrópolis-RJ a perceberam como experiência positiva que contribui para a saúde emocional e gera bem-estar e socialização (SILVA, 2010).

Grupos de artesanato e geração de renda são outras ações psicossociais desenvolvidas na ESF, especialmente pelo ACS, com o apoio do NASF para reabilitação não somente de saúde, mas também de cunho social e econômico. Pesquisa em andamento no LIPAPS tem demonstrado efeito marcante no resgate da autoestima e ressocialização das mulheres das comunidades atendidas (ARAGÃO et al., 2014). Ações que relacionam mente-corpo, tais como atividades físicas e práticas integrativas e complementares (PICS) realizados na ESF em conjunto com o NASF e a Academia da Saúde, ${ }^{3}$ também são efetivas para pessoas com sofrimento difuso e transtornos depressivo-ansiosos, aí se incluindo exercícios, caminhadas, yoga, tai-chi chuan e relaxamento. Dentre as PICS, práticas mindfulness (meditação da atenção plena) vêm alcançando resultados significativos em estudos mundiais na APS (FINUCANE; MERCER, 2006) e começam a ser implementadas no Brasil (DEMARZO et al., 2013).

Adicionalmente, intervenções grupais especializadas também vêm sendo utilizadas na APS para suporte e tratamento de pessoas com transtornos depressivoansiosos mais graves, inclusive vítimas de violência. No Chile, Araya et al. (2003) conduziram estudos sobre intervenções realizadas por enfermeiros e psicólogos para tratamento de depressão moderada a grave, que apresentou melhora e remissão em $70 \%$ dos participantes do estudo randomizado. A adaptação desta intervenção no Brasil incluiu atividades para tratamento de ansiedade, e foi realizada pelo médico 
e enfermeiro da ESF, com apoio de psiquiatra e psicólogo matriciadores. Também teve resultados positivos, com apenas $20 \%$ dos pacientes não apresentando melhora ou remissão, havendo demonstrado que o grupo possibilita uma resposta mais rápida e efetiva no cuidado da depressão, com melhora na qualidade de vida nos domínios psicológicos e dos relacionamentos sociais.

Embora nem todas sejam abordagens psicossociais a serem conduzidas por psiquiatras, devem ser por eles conhecidas e incorporadas em seu arsenal de recomendações terapêuticas por apresentarem forte impacto na SM. Vale lembrar que, no cuidado escalonado, o psiquiatra deve sempre manter-se referência ambulatorial de retaguarda para tratar daqueles que precisem de cuidados mais intensos.

\section{O matriciamento como espaço de cuidado aos transtornos mentais graves e persistentes}

Pessoas com transtornos mentais graves epersistentes (TMGP), que frequentemente apresentam perda significativa de funcionalidade social, têm encontrado na ESF um novo espaço de cuidado, sobretudo quando há o suporte do matriciamento em SM. Alguns estudos baseados em cuidados colaborativos na APS para essa clientela têm demonstrado bons resultados (BERARDI et al., 2014; LEE et al., 2013; SCHOTTLE et al., 2014), mas ainda são poucas as pesquisas realizadas de forma sistemática, segundo revisão de Reilly (2013), que encontrou apenas um ensaio clínico com veteranos norte-americanos com transtorno bipolar do humor de acordo com os critérios utilizados. Neste, o grupo acompanhado por cuidados colaborativos apresentou redução do número de internações e maior qualidade de vida, mas a qualidade da evidência de seus resultados foi considerada baixa ou muito baixa pelo autor. Vale lembrar que há diversas diferenças entre os modelos de cuidados colaborativos analisados e o modelo brasileiro de matriciamento, o que sem dúvida aponta para a necessidade de mais pesquisas sobre o tema.

Com a Reforma Psiquiátrica brasileira, o fechamento dos hospitais psiquiátricos e a implantação dos CAPS, o tratamento dos pacientes foi deslocado para o território onde habitam. A expansão da ESF, que compartilha o cuidado com os CAPS e ambulatórios, fortalece a Reforma Psiquiátrica e contribui para sua consolidação através da ampliação do conceito de saúde-doença, da integralidade da atenção e da participação social, da interdisciplinaridade no cuidado e da territorialização das ações (NUNES, 2007). 
Surge uma estruturação do cuidado às pessoas com TMGP. Todas devem ser acompanhadas pela ESF que coordena a integralidade do cuidado, mas os casos graves que necessitam de atendimento intensivo também estarão nos CAPS, com acesso ao tratamento psicofarmacológico mais complexo e atividades terapêuticas. Aqueles que, mesmo graves, não necessitam dessa intensividade de acompanhamento, irão para ambulatórios especializados. Mas quando estes usuários estão estabilizados e não mais apresentam prejuízo funcional significativo, seu acompanhamento pode ser realizado pela ESF com apoio do psiquiatra. Para que possam desempenhar seu papel no atendimento dessa clientela, é fundamental que os profissionais estejam capacitados e o espaço privilegiado é o da educação permanente promovida pelo matriciamento.

A ESF possui funções importantes na construção do PTS, seja pelo acompanhamento geral da saúde do usuário, seja pelo seu conhecimento da comunidade, da família, da adesão ou não ao tratamento, dos vínculos com cada membro da equipe. Os ACS, em especial, muito podem contribuir para a ampliação da rede de suporte social (LANCETTI, 2000). A ESF é fundamental pela sua facilidade em fazer busca ativa através das visitas domiciliares aos que abandonaram outros serviços e na identificação daqueles que nem procuraram tratamento, alguns mantidos em cárcere privado pelos familiares. Com a expansão da ESF, esses pacientes têm sido identificados, e a partir de intervenções adequadas, novas relações com a família foram estruturadas e a reinserção social promovida, incentivando o acesso a atividades de geração de renda, ao exercício dos direitos e deveres civis, à cultura, à educação, às atividades físicas e de lazer, aos espaços grupais existentes nos dispositivos de cuidado e na comunidade. Para tanto, é necessário enfrentar o estigma da loucura, tanto junto aos pacientes e suas famílias, como nos ambientes coletivos, incluindo a própria ESF. Cabe ao matriciador no matriciamento apresentar-lhes as ferramentas técnicas e estratégias apropriadas para lidar com esses usuários, permitindo mudanças de atitude e diminuição do sentimento de impotência diante desses casos, pela melhoria da efetividade do tratamento e formação de novos padrões vinculares.

No matriciamento, o psiquiatra compartilha algumas ferramentas com bons resultados nos casos em que há dificuldade na adesão ao tratamento. São as medicações injetáveis de depósito, como o haloperidol decanoato, ou a administração da medicação oral supervisionada pela ESF, semelhante ao que é 

tuberculose, que pode ser feita diariamente ou com intervalos maiores de tempo, conforme a necessidade. Tal medida evita a posse de um número grande de comprimidos e a ingestão abusiva ou tentativa de suicídio.

A articulação frequente dos diversos dispositivos da rede é favorecida pelo matriciamento que, através da troca de saberes e informações sobre os usuários, do trabalho com as famílias e diferentes setores da comunidade, promove um cuidado mais integrado. A comunicação dos serviços permite a reavaliação constante dos quadros clínicos e dos projetos terapêuticos: se durante a crise, os usuários necessitam de acompanhamento no CAPS, quando estabilizados, podem permanecer apenas na ESF. Há também usuários que têm indicação para CAPS ou ambulatório, mas não conseguem frequentá-los, e acabam acessando exclusivamente a ESF. Encontros regulares para discussão e consultas conjuntas com toda a equipe são fundamentais na articulação do cuidado desses pacientes e daqueles acompanhados somente pela ESF. Problemas ocorrem quando os profissionais do CAPS contactam apenas os ACS, solicitando auxílio para seus próprios pacientes. Deve-se ressaltar que o matriciamento não deve ocorrer apenas sob demandas pontuais ou urgentes, como frequentemente tem sido observado na prática dos serviços, mas deve ser um processo regular.

Através do matriciamento e do trabalho colaborativo, muito pode ser feito não apenas "por", mas preferencialmente "com" as pessoas com TMGP, na perspectiva da produção de autonomia e da promoção da saúde.

\section{O matriciamento como espaço para o cuidado de usuários com uso nocivo de álcool e/ou drogas}

Os transtornos relacionados ao uso de substâncias constituem um difícil problema de saúde pública, sendo o consumo de álcool e tabaco os mais frequentes. Porém, dada a prevalência e a possibilidade de cuidados, inclusive clínicos pela ESF, apenas o uso problemático de álcool será discutido. Segundo Laranjeira (2007), $3 \%$ dos brasileiros bebem de modo abusivo e $9 \%$ apresentam dependência de álcool, predominando a população masculina. $\mathrm{O}$ consumo de álcool dificulta a adesão a tratamentos de saúde, associa-se à morte e invalidez por causas externas, ao absenteísmo laboral, à violência familiar, negligência e abuso infantil. Na clínica cotidiana, é um problema em que a ESF tem apresentado baixa resolubilidade. 
Contudo, é imperativo ampliar o cuidado. Muitas pessoas com uso problemático de drogas, por episódios repetidos de intoxicação, por abuso ou dependência, não chegam aos serviços especializados, mas podem e devem ser cuidados na sua comunidade. Muitos usuários de drogas não consideram que seu consumo de substâncias configura um problema, mas antes de se conscientizarem, costumam apresentar intercorrências clínicas e seu tratamento pode se tornar um momento precioso para que a ESF aborde o uso de drogas, com apoio do matriciamento com o psiquiatra, ampliando significativamente o escopo de ações de cuidado a nível físico e mental. Quando o consumo de álcool traz prejuízos à pessoa, configurando uso abusivo ou dependência, pode gerar conflitos familiares, que chegam aos profissionais da ESF como demanda de cuidado. Agindo através redução de danos, a díade ESF-psiquiatra pode abordar esses usuários, ampliando sua capacidade crítica, construindo PTS para estas pessoas, mesmo que não queiram abandonar completamente o uso de drogas, buscando o suporte da família, o apoio da rede social e dos recursos comunitários existentes na sua localidade. Nesse processo se constrói também o apoio a estas famílias em seu sofrimento, que muitas vezes também adoecem.

Tratar de transtornos mentais relacionados ao álcool traz o desafio que a integralidade impõe ao raciocínio clínico. Na coleta da história clínica, a avaliação envolve múltiplos sistemas orgânicos e necessita de exame físico criterioso que investigue possíveis lesões e oriente o pedido de exames complementares de maneira racional. $\mathrm{O}$ atendimento conjunto de psiquiatra e generalista permite integrar a abordagem dos problemas físicos e psíquicos e diagnosticar se há transtorno mental de base, que pode indicar que o uso da droga pode ser uma tentativa de autotratamento. O matriciamento instrumentaliza a ESF a utilizar novas tecnologias (exemplo: entrevista motivacional e instrumentos de rastreamento CAGE e AUDIT) e a adotar estratégias de intervenção breve e de desintoxicação ambulatorial (RITSON, 2005; BOURBON, 2012; CHIAVERINI et al., 2011; SMSDC, 2011). Embora mundialmente já se tenha clareza da importância destes recursos na formação do generalista, verifica-se que a graduação em medicina no Brasil pouco contempla esta abordagem.

A elaboração de um PTS é direcionada pelos diagnósticos clínicos e psiquiátricos e envolve aspectos psicossociais. De acordo com a necessidade do paciente, o acompanhamento exige o trabalho em rede, incluindo 
atendimento móvel, emergência, internações em hospitais gerais, atendimento em ambulatório e em CAPS Ad. A atuação no cenário comunitário exige do psiquiatra-matriciador o conhecimento dos fluxos e habilidade de articulação da rede de serviços de saúde de sua área.

A construção de parcerias intersetoriais, que caracterizam o trabalho da APS, demandará também a elaboração de estratégias de prevenção ao uso de drogas em grupos vulneráveis, tais como adolescentes, desempregados e pessoas com sofrimento emocional e transtornos mentais não diagnosticados. Todas essas açôes favorecem o aumento da resolubilidade da assistência e diminuem a lacuna entre a demanda populacional e a oferta de cuidados.

\section{Discussão}

A partir da problematização do que tem sido observado nos projetos de extensão, ensino e pesquisa sobre "Saúde Mental na Atenção Primária" no LIPAPS/ UERJ, e considerando o referencial conceitual dos Cuidados Colaborativos (Apoio Matricial e Matriciamento) e da Atenção Integral à Saúde, entende-se que o psiquiatra tem papel importante no cuidado das demandas de SM que chegam à ESF. Neste sentido, a ESF é um parceiro indispensável, e o território e a comunidade são o novo cenário de atuação do psiquiatra que necessita de novas tecnologias de cuidado para atender as pessoas portadoras de TMC, TMGP, uso nocivo de álcool e as famílias em situação de crise, que são os principais problemas com que se depara no processo de trabalho do matriciamento e nos NASF.

Esse novo cenário impõe transformaçōes para a Psiquiatria. Muda seu local de trabalho (do hospital e serviços especializados para a comunidade e APS), seus agentes (incorpora o médico generalista como agente resolutivo), seu modelo de compreensão (em direção ao modelo biopsicossocial na busca de transcender antagonismos cartesianos), e seu próprio processo de trabalho (que se torna compartilhado, interdisciplinar e integrativo). Assim, agrega novos saberes, trabalha com novos atores e busca disponibilizar o cuidado de SM para todos cidadãos em seus territórios. Por isso, possui grande potencialidade para reduzir a lacuna de cuidado de SM, que é uma prioridade para a Saúde Pública em todo o mundo.

Todas estas alterações demandam um grau equivalente de modificações para formar os atores responsáveis pela Psiquiatria do século XXI. E o caminho para essas transformações já começa a ser traçado nas mudanças das diretrizes 
curriculares 2014, que passaram a considerar a SM como área básica da formação médica, reconhecendo a necessidade de que todo profissional de Medicina seja tão resolutivo em Psiquiatria (Saúde Mental) quanto deve ser na Clínica Geral e Cirúrgica, na Ginecologia e Obstetrícia (Saúde da Mulher), na pediatria (Saúde da Criança) e na Saúde Coletiva. Esse caminho está em constante ampliação na formação continuada dos MFC e onde os temas de SM em geral (onde se incluem a Psicologia Médica e a Psiquiatria, disciplinas tradicionais do curso médico, mas não apenas elas) são frequentes nos congressos da especialidade. Isso sem falar no matriciamento e no estágio nos CAPS, que são parte integrante das residências de MFC. E se desenvolve em todo seu potencial no dia a dia do Apoio Matricial, nas atividades de matriciamento, em que o trabalho interdisciplinar, na comunidade, dentro de uma perspectiva integral, representa o campo ideal para a formação dos médicos para uma Psiquiatria adequada para o século XXI que tenha a superação da lacuna de cuidado como seu objetivo principal.

$\mathrm{O}$ aprendizado para atuar nesta nova realidade é muito mais amplo que o que pode ser ensinado apenas através de programas educativos informativos, que têm falhado em transformar os parâmetros clínicos e a qualidade de vida dos pacientes atendidos, mesmo aumentando a capacidade de detecção e adequando a prescrição medicamentosa dos médicos da APS (GOLDBERG, 1998; GONÇALVES; FORTES; TOFOLI, 2011). Ele necessita de novas metodologias de aprendizagem, novas formas de intervenção, novos modelos de compreensão, novas práticas de apoio mútuo dos profissionais de distintas formações. O processo de matriciamento, enquanto espaço de colaboração e educação permanente, é efetivo na construção dessas alternativas.

A educação permanente é parte estrutural do processo de trabalho no matriciamento, indo além do mero acúmulo de conhecimento, construindo um processo relacional e complexo de intercâmbio multiprofissional que permite melhor interação com a multifacetada realidade humana. Ele realiza suas ações de modo coletivo, em processo que reúne a problematização e a articulação em equipe. Segundo Miccas (2014), esses itens são necessários para a desconstrução do modelo assistencial vigente. Deste modo, a divisão de responsabilidades e a prática da cogestão facilitam a implementação do processo de cuidado no território.

Este artigo apresenta algumas limitações. Em primeiro lugar, não foi possível discutir o trabalho de outros profissionais de SM no matriciamento, por este ser 
extremamente rico para ser abordado em um artigo de tamanho limitado. E, ainda que o foco central tenha recaído sobre a Psiquiatria, algumas áreas desta não foram sequer mencionadas, tais como o cuidado a criança e ao adolescente. Também não se dispõe de pesquisas em número suficiente para fazer deste artigo um artigo de revisão embasado eminentemente em estudos baseados em metodologias científicas mais específicas. Afinal, a implantação do Apoio Matricial varia largamente em todo o país e só agora começa a ser objeto de estudos controlados, incluindo os desenvolvidos pelos LIPAPS/UERJ citados entre as referências. Uma vez que muitos estudos ainda estão em andamento e são parte das atividades de extensão, assistência e ensino universitário, optou-se pela problematização da realidade e sistematização do conhecimento e experiências acumuladas como base no que tem sido observado pelo grupo de pesquisa CNPq "Saúde Mental na Atenção Primária” desde 2002, e pelo LIPAPS/UERJ desde 2010.

\section{Conclusão}

Em todo o mundo, busca-se desenvolver novos modelos e promover a integração de SM na APS. No Brasil, a APS é representada pela ESF e pelos NASF, que atuam conjuntamente através do Apoio Matricial e das atividades de matriciamento. Sendo assim, os protagonistas construtores desse processo são os médicos generalistas, os especialistas em Medicina de Família e Comunidade ou de outras áreas básicas, bem como os psiquiatras e outros profissionais de SM que atuam na APS.

Conclui-se que a APS expande as fronteiras do cuidado de SM e configura um novo desafio para a Psiquiatria século XXI, por ampliar-se dos atendimentos pontuais para a atenção longitudinal; da atuação unidisciplinar para a multi e até mesmo transdisciplinar; da supervisão para o compartilhamento de casos; do foco nos TMPG para a atenção transversal a todas as condições clínicas; do tratamento eminentemente farmacológico para a abordagem biopsicossocial, ou seja, do trabalho especializado para o trabalho em colaboração.

A intenção vital deste artigo foi alimentar - tanto na APS, quanto na SM a discussão crítica sobre o impacto para a Psiquiatria de sua integração com a APS através do cuidado colaborativo (matriciamento) com a ESF. Espera-se que algumas das reflexões aqui apresentadas possam ajudar a Psiquiatria a encontrar as melhores soluções possíveis para reduzir a lacuna assistencial e ampliar o cuidado integral à saúde. ${ }^{5}$ 


\section{Referências}

ANDRADE, L. H. et al. Mental disorders in megacities: findings from the Sao Paulo megacity mental health survey, Brazil. PLoS One, v. 7, n. 2,p. e31879, 2012.

ARAGÃO, E.I.S. et al. Saúde mental na Atenção Primária, apoio a mulheres em grupos de convivência. In: IV CONGRESSO SUL BRASILEIRO DE MEDICINA DE FAMÍLIA E COMUNIDADE. 120 WONCA World Rural Health Conference., 2014, Gramado: Congresso Sul-brasileiro de Medicina de Família e Comunidade, Anais... 2014.

ARAÚJO T. M.; PINHO P. d. S.; ALMEIDA M. M. G. Prevalência de transtornos mentais comuns em mulheres e sua relação com as características sociodemográficas e o trabalho doméstico. Revista Brasileira de Saúde Materno Infantil, v. 5, p. 337-348, 2005.

ARAYA, R. et al. Treating depression in primary care in low-income women in Santiago, Chile: a randomised controlled trial. Lancet, n. 361, p. 995-1000, 2003.

ATHIÉ, K. et al. Matriciamento em saúde mental na Atenção Primária: uma revisão crítica (2000-2010). Revista Brasileira de Medicina de Família e Comunidade, 2013, v.8, n.26, 2013.

BARRETO, A. Terapia Comunitária: passo a passo. 3ed. Fortaleza: Gráfica LCR, 2008.

BARRETO, V.H.L. et al. Tratado de medicina de família e comunidade: princípios, formação e prática. V. 2. Porto Alegre: Artmed, 2012.

BERARDI, D. et al. Primary care psychiatry in Italy. J Nerv Ment Dis., v. 202, n. 6, p. 460-3, June 2014.

BIGRAS, M.; MACHADO, A. da L. Apontamentos e reflexôes sobre programas de apoio familiar que favorecem a competência social da criança. Ciência e Saúde Coletiva. Rio de Janeiro, v. 19, n. 3, mar. 2014.

BONFIM, I. G. et al. Apoio Matricial em Saúde Mental na Atenção Primária à Saúde: uma análise da produção científica e documental. Interface (Botucatu), v. 17, n. 45, p. 287-300. abr./jun. 2013.

BOWER, P. et al. Collaborative care for depression in primary care: making sens of a complex intervention: systematic review and meta-regression. British Journal of Psychiatry, 189:484-493,2006.

BRASIL. Ministério da Educação. Programa de Educação Tutorial (Legislação PET). Portaria no 976, de 27 jul. 2010. Disponível em: <http://portal.mec.gov.br/index. php? option $=$ com_docman $\&$ task=doc_download \&gid $=14912 \&$ Itemid $=484>$. Acesso em: 24 ago. 2014.

BRASIL. Ministério da Educação. Resolução nº 3, de 20 de junho de 2014. Diretrizes Curriculares Nacionais do Curso de Graduação em Medicina. Disponível em: <http:// pesquisa.in.gov.br/imprensa/jsp/visualiza/index.jsp?data $=23 / 06 / 2014$ \&jornal $=1$ \&pagina $=$ 8\&totalArquivos=6>. Acesso em: 03 set. 2014 . 
CAMPOS, G.W.de S. Equipes de referência e apoio especializado matricial: um ensaio sobre a reorganização do trabalho em saúde. Ciência \& Saúde Coletiva. Rio de Janeiro, v. 4, n. 2, p. 393-403, 1999.

CHEW-GRAHAM, C. et al. Aiming to improve the quality of primary mental health care: developing an intervention for underserved communities. BMC Family Practice, 15:68, 2014. CHIAVERINI, D. H. (Org.) et al. Guia prático de matriciamento em saúde mental. Brasília, DF: Ministério da Saúde, 2011.

COELHO, B.M.; ZANETTI, M.V.; LOTUFO NETO, F. Residência em psiquiatria no Brasil: análise crítica. Rev. psiquiatr. Rio Gd. Sul. Porto Alegre, v. 27, n. 1, abr. 2005.

CORREIA C.R.M. Uso de antidepressivos e benzodiazepinicos em mulheres atendidas em unidades de saúde da família e sua dimensão psicossocial. Dissertação (Mestrado em Saúde Coletiva, Epidemiologia) - Instituto de Medicina Social, Universidade do Estado do Rio de Janeiro. Rio de Janeiro, 2014.

COULL, G.; MORRIS, P.G. The clinical effectiveness of CBT-based guided self-help interventions for anxiety and depressive disorders: a systematic review. Psychological Medicine, v. 41, p. 2.239-2252, 2011.

DEMARZO, M.M.P. et al. Mindfulness-based stress reduction (MBSR) in perceived stress and quality of life: an open, uncontrolled study in a Brazilian healthy sample. Explore (New York, N.Y.), v. 10, p. 118-120, 2013.

DEMYTTENAERE, K. et al. Prevalence, severity, and unmet need for treatment of mental disorders in the World Health Organization World Mental Health Surveys. Jama, v. 291, n. 21, p. 2.581-2.590, 2004.

DITTERICH, R.G.; GABARDO M.C.L.; MOYSÉS, S.J. As Ferramentas de Trabalho com Família Utilizadas pelas Equipes de Saúde da Família de Curitiba, PR Saúde Soc. São Paulo, v. 18, n. 3, p.515-524, 2009.

FERREIRA FILHO, M.O. et al A terapia comunitária como estratégia de promoção à saúde mental: o caminho para o empoderamento. Ver. Eletr. Enf., v. 11, n. 4, p. 964-70, 2009.

FINUCANE, A.; MERCER, S.W. An exploratory mixed methods study of the acceptability and effectiveness of mindfulness -based cognitive therapy for patients with active depression and anxiety in primary care. BMC Psychiatry, v. 6, n. 14, 2006.

FONSECA, M.L.G.; GUIMARÃES, M.B.L.; VASCONCELOS, E.M. Sofrimento difuso e transtornos mentais comuns: uma revisão bibliográfica. Rev. APS, v. 11, n. 3, p. 285-294, jul./set. 2008.

FORTES, S.; BALLESTER, D. Saúde mental e Estratégia de Saúde da Família, construção da integralidade, p. 266-270. In: BRASIL, M.A.A. et. al. Psicologia médica, a dimensão psicossocial da prática médica. Rio de Janeiro: Guanabara Koogan, 2012. 
FORTES, S. et al. Transtornos mentais comuns em Petrópolis-RJ: um desafio para a integração da saúde mental com a estratégia de saúde da Revista Brasileira de Psiquiatria, v. 33, n. 2, jun 2011.

FORTES, S.; VILLANO, L.A.; LOPES, C.S. Nosological profile and prevalence of common mental disorders of patients seen at the Family Health Program (FHP) units in Petropolis, Rio de Janeiro. Rev Bras Psiquiatr, v. 30, n. 1, p.32-37, 2008.

GARCIA-HUIDOBRO, D. et al Family intervention to control type 2 diabetes: a controlled clinical trial. Family Practice, v. 28, p. 4-11, 2011.

GASK., L. et al. Improving access to psychosocial interventions for common mental health problems in the United Kingdom: narrative review and development of a conceptual model for complex interventions. BMC Health Services Research, v. 12, n. 249, 2012.

GOLDBERG, D. A bio-social model for common mental disorders. Acta Psychiatr Scand Suppl., v. 385, p. 66-70, 1994.

GONÇALVES, D. A. et al. Brazilian multicentre study of common mental disorders in primary care: rates and related social and demographic factors. Cadernos de Saúde Pública, v. 30, p. 623-632, 2014.

GONÇALVES, D.A. et al. Determinants of common mental disorders detection by general practitioners in primary health care in Brazil. Int J Psychiatry Med., v. 41, n. 1, p. 3-13, 2011. KLEINMAN, A. The illness narratives: suffering, healing and the human condition. New York: Basic Books, 1988.

KNOWLES S.E. et al. Better together? a naturalistic qualitative study of inter-professional working in collaborative care for co-morbid depression and physical health problems. Implement Sci., v. 20, n. 8, p. 110, Sep. 2013.

LANCETTI, A. (Org.). Saúde mental e saúde da família.São Paulo: Hucitec, 2000 (Saúde e Loucura, 7).

LARANJEIRA, R. et al. Levantamento nacional sobre os padrões de consumo de álcool na população brasileira. Brasília: Secretaria Nacional Antidrogas, 2007.

LAVRAS, C. Atenção primária à saúde e a organização de redes regionais de atenção à saúde no Brasil. Saúde Soc., São Paulo, v. 20, n. 4, Dec. 2011.

LEE, S. J. et al. What is needed to deliver collaborative care to address comorbidity more effectively for adults with a severe mental illness? Aust NZ J Psychiatry., v. 47, n. 4, p. 33346, apr. 2013.

MACHADO, D.; CAMATTA, M. Apoio Matricial como ferramenta de articulação entre a saúde mental e a atenção primária à saúde. Cadernos de Saúde Coletiva. Rio de Janeiro, v. 21, n. 2, p. 224-32, 2013. 
MARAGNO, L. et al. Prevalence of common mental disorders in a population covered by the Family Health Program (QUALIS) in São Paulo, Brazil. Cad. Saúde Pública. Rio de Janeiro, v. 22, n. 8, p. 1.639-1.648, ago 2006.

McDANIEL, S.H. et al. Terapia Familiar Médica: um enfoque biopsicossocial às famílias com problemas de saúde. Porto Alegre: Artes Médicas, 1994.

MENEZES, A. L. do A. Intervenções psicossociais para transtornos mentais comuns na Atenção Primária à Saúde. Dissertação (Mestrado em Saúde Coletiva) - Instituto de Medicina Social, Universidade do Estado do Rio de Janeiro. Rio de Janeiro, 2012.

MICCAS, F.L.; BATISTA, S.H.S.da S. Educação permanente em saúde: metassíntese. Rev. Saúde Pública, São Paulo, v.48, n. 1, Feb. 2014.

MINUCHIN, S. Famílias, funcionamento e tratamento. Porto Alegre: Artes Médicas, 1982. NUNES, M.; JUCÁ, V.J.; VALENTIM, C.P.B. Ações de saúde mental no Programa de Saúde da Família: confluências e dissonâncias das práticas com os princípios das reformas psiquiátrica e sanitária. Cadernos de Saúde Pública. Rio de Janeiro, v. 23, n. 10, Oct. 2007.

OLIVEIRA, G.N. Projeto Terapêutico Singular. In: CAMPOS, G.W.S.; GUERRERO, A.V.P. Manual de Práticas da Atenção Básica: saúde ampliada e compartilhada. São Paulo: Hucitec, 2011, p. 283-297.

PINHEIRO, R. Cuidado em saúde. In: Dicionário da Educação Profissional em Saúde, Escola Politécnica Joaquim Venâncio, Fundação Oswaldo Cruz, 2009, s/p. Disponível em: <http://www.epsjv.fiocruz.br/dicionario/verbetes/cuisau.html>. Acesso em: 09 ago 2014.

PORTUGAL, F.B. et al. Sofrimento psíquico e qualidade de vida em pacientes da atenção primária de duas cidades do Brasil. J Bras Psiquiatr., v. 63, n. 1, p. 23-32, 2014.

PRINCE, M. et al. No health without mental health. The Lancet, v. 370, n. 9590, p. 859$877,2007$.

REILLY, S. et al. The role of primary care in service provision for people with severe mental illness in the United Kingdom. PLoS One., v. 7, n. 5, p. e36468, 2012.

RITSON, B. ABC of alcohol. British Medical Journal. n. 330, p.139-141, 2005.

SCHADE N.; TORRES P.; BEYEBACH M. Cost-Efficiency of a Brief Family Intervention for Somatoform Patients in Primary Care Families, Systems, \& Health, v. 29, n. 3, p. 197-205, 2011.

SCHOTTLE, D. et al. Effectiveness of integrated care including therapeutic assertive community treatment in severe schizophrenia spectrum and bipolar disorders: the 24-Month Follow-Up ACCESS II Study. J Clin Psychiatry, Aug 19, 2014. Disponível em: http://www. ncbi.nlm.nih.gov/pubmed/25188752.

SECRETARIA MUNICIPAL DE SAÚDE E DEFESA CIVIL. Superintendência de Atenção Primária. Guia de Referência Rápida. Carteira de Serviços: Relação de serviços prestados na Atenção Primária à Saúde. Rio de Janeiro: SMSDC, 2011. 
depressão: uma estratégia em saúde mental no PSF de Petrópolis. Dissertação (Mestrado) - Programa de Pós-Graduação em Saúde da Família. Universidade Estácio de Sá, Rio de Janeiro, 2010.

STARFIELD, B. Atenção Primária: equilíbrio entre necessidades de saúde, serviços e tecnologia. Brasília: Ministério da Saúde, 2002.

WALSH, F. Fortalecendo a resiliência familiar. Roca: São Paulo, 2005.

WORLD HEALTH ORGANIZATION. Mental Health Atlas. Genebra: WHO, 2005. . Mental Health Atlas. Genebra: WHO, 2011.

World Organization of Family Doctors (WHO/WONCA). Integrating mental health into primary care: a global perspective. Genebra: WHO, 2008.

\section{Notas}

${ }^{1}$ A Declaração Alma-Ata é resultado da Conferência Internacional da Organização das Nações Unidas na URSS.

${ }^{2}$ http://www.globalmentalhealth.org

${ }^{3}$ Programa do Ministério da Saúde, Portarias no 719/2011 e 2.681/2013.

${ }^{4}$ Estratégia DOTS para controle da tuberculose foi pautada em cinco pilares: compromisso político; detecção de casos por baciloscopia; esquemas de tratamento padronizados e tratamento diretamente supervisionado; suprimento regular e ininterrupto dos medicamentos padronizados; e sistema de registro e notificação de casos (WHO, 1991).

${ }^{5} \mathrm{~S}$. Fortes contribuiu com a concepção, conteúdo e redação final de todas as seçôes, revisão crítica do conteúdo intelectual e aprovação da versão final. A. Menezes contribuiu com a redação do conteúdo relacionado às intervenções psicossociais, revisão crítica do conteúdo, edição, revisão geral do texto e aprovação da versão final. K. Athié contribuiu com a redação do conteúdo relacionado à atenção primária, cuidados colaborativos e matriciamento, realizou revisão crítica do conteúdo, revisão geral do texto e aprovação da versão final. L.F. Chazan contribuiu com a redação do conteúdo relacionado à abordagem integral e abordagem familiar, e com a revisão crítica do conteúdo. H. Rocha contribuiu com a redação do conteúdo relacionado aos transtornos por uso de álcool e drogas. J. Thiesen contribuiu com a redação do conteúdo relacionado aos transtornos mentais graves e persistentes. C.R.M. Correia contribuiu com a redação do conteúdo relacionado aos transtornos mentais comuns e a revisão crítica do conteúdo intelectual. T. Pithon contribuiu com a redação do conteúdo relacionado aos transtornos mentais comuns. A. Machado contribuiu com a redação do conteúdo relacionado à abordagem familiar, revisão geral do texto e aprovação da versão final. 
Psychiatry in the $21^{\text {th }}$ century: changes from the integration with Primary Health Care through matrix support

The gap in Mental Health Care is a worldwide problem. Primary Health Care (PHC) is the framework basis for providing integrated care. Integrating Psychiatry into $\mathrm{PHC}$ is fundamental to solve this problem. Based on the issue of how integration of PHC into Mental Health Care is being developed with matrix support from psychiatrists, this paper investigates the role of Psychiatry in the organization of PHC, within a health comprehensive model approach. Problem-based analysis was used to systematize the knowledge and experiences gained by the authors during the execution of several research projects developed at the Laboratory of Interdisciplinary Research in Primary Health Care (LIPAPS) of Rio de Janeiro State University. Conceptual references such as Comprehensive Care, Collaborative Care and the Brazilian Model of Matrix Support Teams, as well as the most frequent Mental Health demands in PHC are reviewed, while discussing their influence on mental health care, medical education and changes imposed on Psychiatry. In conclusion, PHC may be considered as a new scenario for Psychiatry that raises important challenges related to mental health education and services.

> Key words: Mental Health; Psychiatry; Primary Care; matrix support teams; comprehensive health care. 